\title{
Autonomia e Vulnerabilidade do Sujeito da Pesquisa
}

\author{
AUTONOMY AND VULNERABILITY OF SUBJECT \\ IN CIENTIFIC RESEARCH
}

\author{
Cristiana Tengan $(*)$ \\ Paulo César Venancio (**) \\ Fernanda Klein Marcondes (***) \\ Pedro Luis Rosalen (****)
}

\section{RESUMO}

A partir do fim da Segunda Guerra Mundial passaram a ser elaboradas regulamentações internacionais para o desenvolvimento de Pesquisas envolvendo seres humanos. Para a realização destas pesquisas é essencial que princípios éticos tais como autonomia e vulnerabilidade do sujeito da pesquisa sejam respeitados. Considerando a importância do conhecimento dos pesquisadores sobre os aspectos éticos envolvidos na pesquisa com seres humanos, o presente estudo tem como objetivo discutir estes aspectos dentro de um contexto histórico. Nos dias atuais, pesquisas incluindo grupos vulneráveis e pessoas com autonomia reduzida são comumente realizadas $e$, apesar da existência de normas internacionais e nacionais, além de códigos e diretrizes publicadas a este respeito, muitas destas são realizadas desrespeitando a ética e os princípios morais, ferindo a dignidade humana.

\section{Palavras-chave}

Bioética; Autonomia Pessoal; Vulnerabilidade.

(") Cirurgiā-Dentista, Mestre em Cariologia FOP/UNICAMP.E-mail: cristengan@fop.unicamp.br

$\left(^{* *}\right)$ Farmacêutico, Professor do Colégio Técnico de Limeira-CotilUNICAMP. E-mail: pcvenancio@ponkan. cotil.unicamp.br

("**) Professora-Associada da Faculdade de Odontologia de Piracicaba/UNICAMP. E-mail: fklein@fop.unicamp.br

("***) Professor-Doutor do Programa de Pós-Graduação em Odontologia da Faculdade de Odontologia de Piracicaba-FOP/UNICAMP. E-mail: rosalen@fop.br

Recebido: 19.6.05. Aprovado: 23.8.05 


\section{ABSTRACT}

After the Second World War, international regulations started to be elaborated to the research development involving the human being. It's essential that some ethnic principles such as autonomy and vulnerability of the person can be respected to the development of researches in human. Considering the importance of the researchers knowledge over the ethnics aspects involved into research with human being, the current study purposes to discuss these aspects in a historical context. Nowadays, some researches including vulnerable groups and people with reduced autonomy are normally achieved. Despite the existence of international and national norms, code and directions published regarding them, a lot of these researches are achieved without respecting the ethnical and moral principles that offend the human dignity.

\section{Key words}

Bioethics; Personal Autonomy; Vulnerability.

\section{INTRODUÇÃO}

A partir do final da Segunda Guerra Mundial, passaram a ser elaboradas as regulamentações internacionais para o desenvolvimento de Pesquisas envolvendo seres humanos. O Código de Nuremberg foi o primeiro documento criado neste sentido e, também, o primeiro a enunciar a prerrogativa de respeito à autonomia dos sujeitos da pesquisa. A autonomia é um dos referenciais básicos da Bioética, segundo o qual cada indivíduo é capaz de deliberar e tomar decisões, de acordo com o seu plano de vida e ação(1). Segundo as ciências jurídicas, o conceito de autonomia refere-se "à pessoa maior de idade capaz de decidir livremente sobre questóes da sua vida ou de seus dependentes e, conseqüentemente, suportar as decorrências de sua decisão"(2). O princípio da autonomia tem a sua expressão no documento denominado "consentimento informado", desde que haja o entendimento da informação transmitida ${ }^{(3)}$. Portanto, sua linguagem deve ser simples e de fácil

(1) LOWDEN, J. Children's rights: a decade of dispute. Journal of Advanced Nursing, v. 37, n. 1, p. $100-$ 107, 2002, HARDY, E.; BENTO, S. F.; OSIS, M. J. D. Consentimento livre e esclarecido: experiência de pesquisadores brasileiros na área da regulação da fecundidade. Caderno de Saúde Pública, v. 20, n. 1 , p. 216-223, 2004.

(2) GUIMARĀES, M. C. S.; NOVAES, S. C. Autonomia reduzida e vulnerabilidade: liberdade de decisão, diferença e desigualdade. 2005. Disponivel em: <http://www.portalmedico.org.br/revista/bio1v7/ autonomia.htm. Acesso em: 6.abr.2005.

(3) ROGER, B. Research with protected population - vulnerable participants. Journal AAOHN, v. 53, n. 4, p. 156-157, 2005. 
entendimento. Muitas vezes, grupos como, idosos, pacientes psiquiátricos e pessoas com baixo grau de instrução apresentam um grau de entendimento reduzido, requerendo um termo com uma linguagem mais fácil, ou a presença de um responsável pela pesquisa, para que a autonomia da mesma seja resguardada ${ }^{(4)}$.

Outro termo que merece consideração é a "vulnerabilidade" do sujeito da pesquisa. A Resolução n. 196/96, do CNS, que estabelece as normas para Pesquisa com Seres Humanos no Brasil, define vulnerabilidade na seção II.15 como "estado de pessoas ou grupos que, por quaisquer razões ou motivos, tenham a sua capacidade de autodeterminação reduzida, sobretudo no que se refere ao consentimento livre e esclarecido" e, recomenda que a observância dos princípios éticos, em pesquisa, implica em "proteção aos grupos vulneráveis", devendo tratá-los em sua dignidade, respeitá-los em sua autonomia e defendê-los em sua vulnerabilidade. A vulnerabilidade é função de uma relação social, cultural, política e econômica desigual e, como conseqüência de uma relação de desigualdade, pode manifestar-se de modo individual ou coletivo, manifestar-se entre indivíduos, entre diferentes grupos, culturas ou etnias minoritárias em relação a um grupo mais amplo, ou mesmo, entre países ${ }^{(5)}$. Portanto, são exemplos de grupos vulneráveis, muIheres grávidas ou não, crianças, prisioneiros, refugiados, pessoas pobres e minorias étnicas ${ }^{(6)}$.

Considerando a importância do conhecimento dos princípios de autonomia e vulnerabilidade do sujeito da pesquisa pelos pesquisadores, o presente estudo tem como objetivo discutir estes princípios dentro de um contexto histórico.

\section{HISTÓRICO}

Após o término da Segunda Guerra Mundial, juízes dos Estados Unidos reuniram-se para julgar os crimes cometidos por médicos nazistas em campos de concentração. Este fato, noticiado mundialmente, devido às atrocidades cometidas em nome da ciência, resultou na elaboração do Código de Nuremberg ${ }^{(7)}$.

Apesar do conhecimento dos horrores acontecidos nos campos de concentração e da elaboração do Código de Nuremberg, as pesquisas com

(4) GLOCK, R. S.; GOLDIM, J. R. Informed consent in gerontology. Eubios Journal of Asian and International Bioethics, v. 13, p. 6-8, 2003.

(5) ZOBILI, E. L. C. P.; FRACOLLI, L. A. Vulnerabilidade do sujeito de pesquisa. Caderno de Ética em Pesquisa, n. 8, p. 20-21, 2001.

(6) LOTT J. P. Module three: vulnerable/special participant population. Developing World Bioeth, v. 5, n. 1, p. 30-54, 2005.

(7) DINIZ, D.; CORREAA, M. Declaração de Helsinki: relativismo e vulnerabilidade. Caderno de Saúde Pública, v. 17, n. 3, p. 679-688, 2001. 
seres humıanos que feriam os princípios éticos continuaram a ser realizadas. Nos anos 60, o caso da Talidomida, uma pesquisa que ficou mundialmenie conhecida na qual mais de 20 mil mulheres, dentre elas 3.750 em idade reprodutiva, foram submetidas a testes de eficácia da droga nos Estados Unidos, sem sequer saberem que estavam participando de experimentos. Devido a inúmeros casos como este, no ano de 1964, foi elaborada pela Associação Médica Mundial, a Declaração de Helsinki, estabelecendo a obrigatoriedade da obtenção do termo de consentimento informado voluntário, por escrito, de cada pessoa que participa de um estudo(8). Esta declaração é revisada periodicamente, sendo a última em $2002^{(9)}$. Entretanto, denúncias de diversos casos de manipulação usando enfermos social e mentalmente fragilizados como sujeitos de experimentação, vieram ao conhecimento público no início dos anos 70 , e resultaram na elaboração do Relatório de Belmont. A grande inovação deste relatório foi o estabelecimento da necessidade de se observar e respeitar, além do princípio da autonomia (necessidade de consentimento informado), também os princípios da beneficência (atenção aos riscos e benefícios), não maleficiência e justiça (eqüidade quanto aos sujeitos de experimentação) ${ }^{(10)}$.

O Brasil não foi indiferente às questões éticas relacionadas às pesquisas com seres humanos. Em 1978, a Câmara Técnica de Medicamentos do Conselho Nacional de Saúde, do Ministério da Saúde, publicou a Resolução Normativa 1/78, referente aos aspectos éticos da experimentação terapêutica, que afirmava que cabia ao pesquisador decidir se o consentimento do paciente deveria ser oral ou por escrito. Em 1981, a Divisão Nacional da Vigilância Sanitária de Medicamentos do Ministério da Saúde, preparou um documento padrão denominado de "Termo de Conhecimento de Risco", que deveria ser utilizado em ensaios de medicamentos; mas, este foi ignorado pelos pesquisadores. Posteriormente, em 1987, o Conselho Federal de Medicina, elaborou um novo Código de Ética Médica, que estabeleceu que, para a realização de pesquisas com seres humanos, deveria ser obtido um consentimento por escrito, após esclarecer os sujeitos acerca da natureza e conseqüência da pesquisa. Em 1988, o Conselho Nacional de Saúde, estabeleceu o consentimento informado, como uma concordância por escrito, pela qual o sujeito da pesquisa (ou seu representante legal) aceita participar da pesquisa, estando totalmente informado sobre os procedimentos e riscos, com total independência, para concordar ou não, em participar, livre de qualquer forma de coerção. Entretanto, estudos considerados de risco (por exemplo, estudos prospectivos que obtêm dados por meio de procedimentos de diagnóstico físico ou psicológico usuais ou tratamentos

(8) Id. Ibid.

(9) RIIS, P. Thirty years of bioethics: the Helsinki Declaration 1964-2003. New Rev. Bioeth., v. 1, n. 1, p. 15-25, 2003.

(10) JUNGES, J. R. Ética e consentimento informado. Caderno de Ética em Pesquisa, n. 4, p. 22-23, 2000. 
de rotina), poderiam ter somente o consentimento verbal e, se o estudo fosse considerado sem risco (como por exemplo, entrevistas e revisão de dados clínicos), a pesquisa poderia ser conduzida sem o consentimento informado(11).

Apesar de representar um avanço na legislação brasileira, a Resolução n. 1/88 era muito restrita à área médica e de medicamentos, detalhista, com característica cartorial e com pouca reflexão ética e, devido à evolução científica e tecnológica da época, precisou ser revista. Assim, o Conselho Nacional de Saúde, juntamente com representantes da sociedade civil e comunidade científica, apresentaram uma proposta de resolução, com força de Lei, que culminaram com a revogação da Resolução n. 1/88 e, com a aprovação da Resolução CNS n. 196/96 de 10 de outubro de 1996, sobre pesquisas envolvendo Seres Humanos ${ }^{(12)}$. Esta Resolução é inovadora e avançada por constituir um controle social na pesquisa e, desta forma, tem sido indicada como modelo a ser seguido por outros países que ainda não possuem normas para pesquisas com seres humanos ${ }^{(13)}$.

Porém, mesmo após a aprovação da Resolução CNS n. 196/96 ${ }^{(14)}$, pesquisas clínicas continuam sendo feitas de maneira abusiva. Em julho de 2000, na favela Heliópolis, zona Sul de São Paulo, vinte voluntários da pesquisa foram submetidos a diversas aplicaçōes de laser de forma intravenosa, supostamente eficaz no tratamento de patologias como asma, úlcera varicosa e outras doenças inflamatórias. Inúmeras irregularidades foram constatadas: ausência de um protocolo de pesquisa, Termo de Consentimento Livre e Esclarecido (TCLE), desinformação do sujeito da pesquisa com relação aos riscos e efeitos colaterais e falta de registro do equipamento de laser junto à Agência Nacional de Vigilância Sanitária (ANVISA). Por estas irregularidades, a pesquisa foi interrompida. Porém, após sua interrupção, os voluntários revoltaram-se contra o Conselho de Medicina, a Vigilância Sanitária e jornais, pois consideraram que haviam perdido uma opção de tratamento para suas enfermidades. Portanto, observa-se que embora estes voluntários tenham sido "aproveitados" como sujeitos de uma pesquisa oportunista, a exclusão social e a dificuldade de acesso ao atendimento no sistema público de saúde, podem conduzir o indivíduo a um estado de maior vulnerabilidade, dificultando o exercício pleno da autonomia do sujeito da pesquisa ${ }^{(15)}$.

(11) HARDY, E.; BENTO, S. F.; OSIS, M. J. D. Consentimento livre e esclarecido: experiência de pesquisadores brasileiros na área da regulação da fecundidade, cit.

(12) BRASIL. Conselho Nacional de Saúde. Resolução n. 196/96. Disponível em: <http:// www.conselho.saude.gov. br/docs/Reso 196.doc. bioetica. Acesso em: 29.jun.2005.

(13) HARDY, E.; BENTO, S. F.; OSIS, M. J. D. Consentimento livre e esclarecido: experiência de pesquisadores brasileiros na área da regulação da fecundidade, cit.

(14) BRASIL. Conselho Nacional de Saúde. Resoluçāo n. 196/96, cit.

(15) ESTUDO em favela ignora ética. Caderno de Etica em Pesquisa, n. 5, p. 15-16, 2000. 


\section{PACIENTES PSIQUIÁTRICOS}

O paciente psiquiátrico é considerado como um paciente como outro qualquer ${ }^{(16)}$, embora constitua um grupo vulnerável ao abuso(17). O Código de Nuremberg estabeleceu a necessidade do consentimento voluntário do próprio paciente, sendo imprescindível que, para cada paciente psiquiátrico candidato a participar da pesquisa, se estabeleça o grau de capacidade de expressar o consentimento livre e esclarecido, avaliado por profissional psiquiatra e que não seja envolvido no projeto. No caso de drogas, com ação psicofarmacológica, deve ser feita análise crítica quanto aos riscos eventuais de se criar dependências ${ }^{(18)}$. Caso o paciente se manifeste contrário à pesquisa, expressando-se de qualquer forma, a vontade dele deverá ser respeitada(19). A Convenção sobre os Direitos Humanos e Pesquisa Biomédica, do Conselho da Europa, de $1997 \mathrm{diz}$ que, em caso de participantes com problemas psicológicos, impossibilitados de dar o seu consentimento, a pesquisa só poderá ser realizada sempre que trouxer benefício ao próprio paciente ${ }^{(20)}$.

\section{POPULAÇÕES INDÍGENAS}

As tribos indígenas são, antropologicamente, diferentes devido à cultura, tradição e práticas peculiares. Ao longo dos anos, o deslocamento e o contacto com outras culturas trouxeram mudanças socioculturais e de valores. Devido à pobre infra-estrutura de saúde, alto nível de pobreza e ignorância, estas comunidades são consideradas altamente vulneráveis(21).

Muitos abusos foram realizados, como o relatado na década de 60 , quando ocorreu, na Universidade de Michigan, uma possível contaminação proposital de populações de indígenas lanomâmis com o microrganismo Edmonson B. Este microrganismo gera sintomas semelhantes aos do sarampo. Ele teria sido inoculado, nestas populações, pela equipe do geneticista norte-americano James Neel, em uma campanha de vacinação. As populações lanomâmis, envolvidas estavam localizadas na Venezuela e no Brasil. O objetivo desta pesquisa seria estudar as reações destas populações, nesta época, com pouco contato com outras comunidades, quando da ocorrência de uma doença desconhecida e contagiosa. Durante o decorrer

(16) SEGRE, M. Autonomia e pacientes psiquiátricos. Caderno de Ética em Pesquisa, n. 3, p. 12, 1999. (17) SCHÜKLENK, U. Protecting the vulnerable: testing time for clinical research ethics. Social Science \& Medicine, v. 51, p. 969-977, 2000.

(18) HOSSNE, W. S. Autonomia e pacientes psiquiátricos. Caderno de Ética em Pesquisa, n. 3, p. 12, 1999.

(19) BONTEMPO, C. Autonomia e pacientes psiquiátricos. Caderno de Ética em Pesquisa, n. 3, p. 12, 1999.

(20) CLOTET, J. Autonomia e pacientes psiquiátricos. Caderno de Ética em Pesquisa, n. 3, p. 12, 1999.

(21) NAIK, E.; KARPUR, A.; TAYLOR, R. et al. Rural indian tribal communities: an emerging high-risk group for HIVIAIDS. International Health and Human Rights, v. 5, n. 1, 2005. Disponivel em: <http:/l www.biomedcentral.com/1472-698X/5/1>. Acesso em: 8.jun.2005. 
desta pesquisa, os pesquisadores, teriam recebido uma ordem de não tratar os indígenas doentes, mas apenas observar as suas reações. Vários indígenas teriam morrido em conseqüência desta contaminação. ${ }^{(22)}$

Um outro fato ocorreu no início dos anos 80 , quando o chefe da comunidade indígena Ucluelet autorizou a utilização de amostras, de seu sangue, para pesquisa sobre artrite, cujo objetivo era avaliar aspectos genéticos associados às doenças reumatológicas. O geneticista responsável coletou sangue de outros 900 indígenas. No final da década, o geneticista mudou-se de país (do Canadá para os Estados Unidos), levando consigo todas as amostras de sangue. Desde a mudança do geneticista, os indígenas não obtiveram resposta sobre o que havia sido feito com o material biológico estocado. Em 2000, ficaram sabendo que o pesquisador havia utilizado este mesmo material biológico para outros estudos, em diferentes locais do mundo, sem ter solicitado novo consentimento. Atualmente, o pesquisador é diretor do Instituto de Antropologia Biológica da Universidade de Oxford, na Inglaterra. O conselho tribal reuniu-se, no final de 2000, para solicitar a devolução das amostras, para evitar outros usos indevidos ${ }^{(23)}$.

Somente em 2000, a preocupação com a vulnerabilidade do índio e da sua cultura e o interesse crescente em pesquisas com esta população, muitas delas isoladas, fizeram com que esta área obtivesse maior atenção com a elaboração da Resolução CNS n. 304/2000 que garantiu o respeito aos aspectos culturais peculiares, não admitindo a exploração das comunidades indígenas ${ }^{(24)}$.

\section{MULHERES}

No final da década de 80 , início da década de 90 , debates foram realizados na área de fecundidade, onde grupos organizados de mulheres, em defesa dos direitos reprodutivos, denunciaram pesquisas realizadas na área de fecundidade. Os pesquisadores tiravam vantagens de muIheres pobres e ignorantes sobre o assunto estudado, que, sem o poder de argüir ou fazer perguntas, assinavam o termo de consentimento, confiando nos pesquisadores. Pesquisas clínicas realizadas com contraceptivo foram testadas tanto em países desenvolvidos, como em desenvolvimento; mas, quando se utilizavam novas drogas, estas eram primeiramente testadas

(22) GOLDIM, J. R. Contaminação proposital em populações ianomâmis. 2001. Disponivel em: <http:// www.bioetica.ufrgs.br/sarampo.htm>. Acesso em: 10.maio.2005.

(23) /d. Uso de amostra de sangue sem permissão em indígenas canadenses. 2001. Disponível em: <http://www.bioetica.ufrgs.br/canada.htm>. Acesso em: 10.maio.2005.

(24) MELO, A. C. R.; LIMA, V. M. Bioética: pesquisa em seres humanos e comitês de ética em pesquisa. Breves esclarecimentos. Rev. Digital, n. 78, 2004. Disponivel em: <http://www.efdesportes.com/efd78/ etica.htm>. Acesso em: 6.abr.2005. 
em países em desenvolvimento, com pessoas pobres, sem instrução e sem o consentimento da voluntária ${ }^{(25)}$.

Sendo assim, a mulher deve ser vista como um grupo que sofre desvantagens sociais (com relação à aceitação da maternidade como uma condição natural, e pressões sociais para a procriação) e morais (falta de poder nas relaçōes afetivo-sexuais) ${ }^{(26)}$ devendo ser considerada como pertencente a um grupo vulnerável, merecendo cuidados especiais.

\section{POPULAÇÓES CARENTES}

Outro grupo que passou a ser alvo, para experimentos de alto risco, foi o de populações carentes. Estas caracterizam-se como grupo vulnerável pela restrição imposta pelo poder hierárquico a que estão submetidas e pela possibilidade de receberem algum rendimento.

Em países em desenvolvimento, como o Brasil, o acesso aos serviços de saúde e aos recursos médicos muitas vezes é facilitado para o sujeito que está participando de um estudo, podendo funcionar como uma forma de pressão para as pessoas participarem de uma pesquisa. Assim, apesar de não haver coação, muitas vezes os pacientes sentem-se obrigados a participar da pesquisa pela própria condição de vulnerabilidade social em que se encontram ${ }^{(27)}$.

Recentemente, 100 fazendeiros infectados por HIV, via sangue contaminado na década de 90 , foram recrutados para uma pesquisa clínica na China, no qual a droga testada seria a VGV-1. Os voluntários não foram informados sobre efeitos colaterais, assinaram um consentimento informado que não entenderam, não foram informados sobre os resultados da pesquisa e não tiveram as suas despesas ressarcidas.

O crescente interesse dos pesquisadores em trabalhar na China é decorrente das grandes oportunidades clínicas e de expansão de mercado. Além disso, diferentemente dos países ocidentais, não há regras quanto à maneira de recrutamento dos voluntários ou exigência do consentimento informado(28).

\section{CRIANÇAS, JOVENS E ADOLESCENTES}

Potencialmente, a criança é autônoma; entretanto, para que possa consentir com o tratamento ou cuidados recebidos, deverá receber informações

(25) HARDY, E.; BENTO, S. F.; OSIS, M. J. D. Consentimento informado normatizado pela Resolução 196/96: conhecimento e opinião de pesquisadores brasileiros. Rev. Bras. de ginecologia e obstetricia, v. 24 , n. 1,p. 59-65, 2002.

(26) GUILHERM, D. New reproductive Technologies, ethics and legislation in Brazil: a delayed debate. Bioethics, v. 15, n. 3, p. 218-230, 2001.

(27) HARDY, E.; BENTO, S. F.; OSIS, M. J. D. Consentimento livre e esclarecido: experiência de pesquisadores brasileiros na área da regulação da fecundidade, cit.

(28) CYRANOSKI, D. Consenting adults? Not necessarily ... Nature, v. 435, p. 138-139, 2005. 
relevantes, explicações, ter tempo para questionamento e discussōes, a fim de que possa desenvolver conhecimentos relevantes ${ }^{(29)}$. No Simpósio Internacional sobre Bioética e Direito das Crianças, realizado em 2000, foi estabelecido que a criança deve participar das decisões relativas a sua saúde e educação à medida que a sua autonomia se afirmar. Quando houver diferenças de interesses, o interesse da criança deve prevalecer sobre o do adulto. Ou seja, caso a criança recuse participar de uma pesquisa, sua decisão deverá ser respeitada(30).

Segundo Rossi(31), nos Estados Unidos da América, a autorização dos pais protege as crianças de possíveis riscos, enquanto que o consentimento da criança demonstra respeito a ela e ao desenvolvimento da sua autonomia.

A questão da menoridade está no Código Civil Brasileiro como abaixo de 21 anos, existindo, porém, algumas situações de emancipação. O Estatuto da Criança e Adolescente ressalta certa autonomia do adolescente quanto aos seus interesses. Cabe ao Código de Execução Penal analisar eticamente a questão e, definir a necessidade de solicitar autorização aos pais ou responsáveis legais, existindo ainda a possibilidade de buscar autorização no Ministério Público, quando não for de interesse do menor, o contacto com os seus pais.

Com relação à compensação financeira, esta deve ser bem estabelecida, principalmente em relação aos grupos vulneráveis, como crianças e pacientes mentalmente incompetentes, pois muitas vezes, os pais ou guardiōes são responsáveis pelo recebimento do benefício financeiro e, motivados por ganhos em pesquisas, envolvem as suas crianças em experimentos. ${ }^{(32)}$

\section{IDOSOS}

Pesquisas realizadas com pacientes idosos também devem preservar a sua autonomia, mesmo se estes se encontram em situações de incapacidade temporária ou definitiva. Seus desejos ou restrições de tratamento devem ser respeitados. O fundamental é reconhecer que o fato de ser idoso não impede o indivíduo de tomar suas decisões e exercer a sua vontade pessoal, baseado em seus valores ${ }^{(33)}$.

(29) LOWDEN, J. op. cit.

(30) BIOÉTICA e os direitos da criança. Caderno de Ética em Pesquisa, n. 5, p. 9, 2000.

(31) ROSSI, W.C.; REYNOLDS, W.; NELSON, R. M. Child assent parental permission in pediatric research. Theor. Med. Bioeth., v. 24 n. 2 p. 131-148, 2003.

(32) RESNIK, D. B. Research participation and financial inducements. The American Joumal of Bioethics, v. 1, n. 2, p. 54-56, 2001.

(33) GLOCK, R. S.; GOLDIM, J. R. op. cit. 
Por meio do consentimento informado, o paciente é capaz de exercer a sua autonomia. Estudiosos ${ }^{(34)}$ verificaram o uso e adequação do consentimento informado em pacientes idosos e concluíram que a maioria considera os termos difíceis de entender, aceitando participar da pesquisa previamente à leitura do termo. Os autores sugerem que maior atenção deva ser dada à redação dos termos, principalmente para este grupo.

Sendo assim, o desafio para o bioeticista é identificar falhas no processo de consentimento, resguardando a autonomia do idoso, independentemente da fragilidade física, reduzida privacidade e capacidade de decisão, prejudicada por alguma doença neurológica ${ }^{(35)}$.

\section{DETENTOS, PRESIDIÁRIOS OU PRISIONEIROS}

Os detentos, presidiários ou prisioneiros devem merecer igual consideração aos demais voluntários de pesquisas. Toda a exigência deve ser atendida e devem ser especialmente verificados os aspectos que restrinjam o seu consentimento voluntário, pois a pessoa que já foi condenada, poderá ser novamente exposta a riscos elevados, justamente por estar encarcerado. Entretanto, proteger um grupo, sabidamente vulnerável, pela exclusão da sua participação em projetos de pesquisa, poderá ser uma forma de proteção discriminatória. Ao impedir a participação deste grupo em pesquisas, o pesquisador poderá impedir o acesso a um benefício potencial. Sendo assim, recomenda-se que em pesquisas clínicas, onde os participantes tenham benefícios pessoais e diretos com o estudo, os prisioneiros também possam ser convidados a participar, com algumas salvaguardas que garantam a sua voluntariedade ${ }^{(36)}$.

O primeiro relato de pesquisas abusivas com este grupo ocorreu em 1721, quando foi testado, em 6 prisioneiros, o método grego de evitar a varíola que consistia em realizar desenhos cruciformes nas bochechas e no queixo das pessoas com uma agulha molhada no líquido das lesōes de uma pessoa infectada. Embora, leis de proteção a este grupo vulnerável tenham sido criadas, abusos continuaram a serem relatados, como o caso ocorrido em 1972 , em que $90 \%$ das pesquisas farmacológicas de fase I, isto é, do uso de drogas experimentais, eram realizadas em presidiários ${ }^{(37)}$.

Historicamente, pôde-se verificar que o Código de Nuremberg (1947) foi omisso com a questão dos prisioneiros. Posteriormente, em 1952, a Asso-

(34) GLOCK, R. S.; GOLDIM, J. R. op. cit.

(35) Id. Ibid.

(36) GOLDIM, J. R. Pesquisas em prisioneiros. 2001. Disponivel em: <http://www.bioetica.ufrgs.br/ pesqpris.htm>. Acesso em: 10.maio.2005.

(37) Id. Ibid. 
ciação Médica Americana (AMA), elaborou uma resolução contra a participação de presidiários em projetos de pesquisa, não com o objetivo de proteger os presos contra eventuais abusos, mas sim evitar que condenados tivessem acesso à liberdade condicional, por terem participado de projetos de pesquisa. A primeira edição das diretrizes internacionais, elaborada pelo Conselho das Organizaçōes Internacionais de Ciências Médicas (CIOMS), em 1983, abordava a questão da utilização dos presidiários em um dos seus itens. Neste mesmo ano, a AMA propôs a proibição de pesquisa com prisioneiros civis e militares, assim como com populaçōes de zonas ocupadas. A segunda edição do CIOMS, em 1993, na Diretriz n. 7, impede a discriminação dos presidiários quanto ao acesso a novos recursos terapêuticos. No Brasil, as normas para a pesquisa em Seres Humanos (Resolução n. 196/96) estabelecem, em seu item IV.3.b, que todos os participantes devem ter assegurada a garantia da liberdade em consentir. $O$ fato de estar aprisionado pode interferir nesta característica, influenciando a voluntariedade devido a características de pertencer, neste momento, a um grupo vulnerável. $\mathrm{E}$, em outubro de 2002, o órgão encarregado de proteger os participantes de pesquisa nos Estados Unidos, OHRP, lançou um documento indicando a possibilidade de aprovar pesquisas epidemiológicas, realizadas em população carcerária, permitindo conhecer a realidade deste grupo e estabelecendo políticas para este grupo ${ }^{(38)}$.

\section{OUTROS GRUPOS VULNERÁVEIS}

Um outro grupo vulnerável à exploração e ao abuso são os indivíduos submetidos a situaçōes de hierarquia, ou seja, dependência ou situação desigual, como os militares e estudantes. Este grupo constitui uma categoria de pessoas que vive uma relação desigual de poder, entre o possível sujeito da pesquisa com o que tenta convencê-lo a aderir ao projeto de pesquisa. Considerando que nestes casos existe uma grande possibilidade de abuso, o consentimento informado é extremamente importante e tem a finalidade de resguardar a autonomia do indivíduo que vive esta relação de dependência ${ }^{(39)}$.

\section{CONCLUSÃO}

Mais de trinta anos passaram-se desde que a Declaração de Helsinki foi elaborada e, até hoje, pesquisas realizadas com seres humanos ainda ferem os princípios éticos e morais, principalmente quando envolvem grupos

(38)GOLDIM, J. R. Pesquisas em prisioneiros, cit.

(39) SCHÜKLENK, U. op. cit. 
vulneráveis. Portanto, com a finalidade de resguardar a autonomia do voluntário da pesquisa, o Termo de Consentimento Livre e Esclarecido é um documento essencial e deve apresentar uma linguagem simples e de fácil entendimento.

\section{REFERÊNCIAS}

BIOÉTICA e os direitos da criança. Caderno de Ética em Pesquisa, n. 5, p. 9, 2000.

BONTEMPO, C. Autonomia e pacientes psiquiátricos. Caderno de Ética em Pesquisa, n. 3, p. 12, 1999.

BRASIL. Conselho Nacional de Saúde. Resolução n. 196/96. Disponível em: <http://www.conselho.saude.gov. br/docs/Reso 196.doc. bioetica. Acesso em: 29 jun. 2005.

CLOTET, J. Autonomia e pacientes psiquiátricos. Caderno de Ética em Pesquisa, n. 3, p. 12, 1999.

CYRANOSKI, D. Consenting adults? Not necessarily ... Nature, v. 435, p. 138139, 2005.

DINIZ, D.; CORREAA, M. Declaração de Helsinki: relativismo e vulnerabilidade. Caderno Saúde Pública, v. 17, n. 3, p. 679-688, 2001.

ESTUDO em favela ignora ética. Caderno de Ética em Pesquisa, n. 5, p. 1516, 2000.

GLOCK, R. S.; GOLDIM, J. R. Informed consent in gerontology. Eubios Journal of Asian and International Bioethics, v. 13, p. 6-8, 2003.

GOLDIM, J. R. Contaminação proposital em populações ianomâmis. 2001. Disponível em: <http://www.bioetica.ufrgs.br/sarampo.htm>. Acesso em: 10. maio.2005.

. Pesquisas em prisioneiros. 2001. Disponível em: <http://www.bioetica. ufrgs.br/pesqpris.htm>. Acesso em: 10.maio.2005.

. Uso de amostra de sangue sem permissão em indígenas canadenses. 2001. Disponível em: <http://www.bioetica.ufrgs.br/canada.htm>. Acesso em: 10.maio.2005.

GUILHERM, D. New reproductive Technologies, ethics and legislation in Brazil: a delayed debate. Bioethics, v. 15, n. 3, p. 218-230, 2001.

GUIMARÃES, M. C. S.; NOVAES, S. C. Autonomia reduzida e vulnerabilidade: liberdade de decisão, diferença e desigualdade. 2005. Disponível em: <http://www.portalmedico.org.br/revista/bio1v7/autonomia.htm>. Acesso em: 6.abr.2005. 
HARDY, E.; BENTO, S. F.; OSIS, M. J. D. Consentimento informado normatizado pela Resolução n. 196/96: conhecimento e opinião de pesquisadores brasileiros. Rev. Bras. de ginecologia e obstetrícia, v. 24, n. 1, p. 59-65, 2002.

. Consentimento livre e esclarecido: experiência de pesquisadores brasileiros na área da regulação da fecundidade. Caderno Saúde Pública, v. 20, n. 1, p. 216-223, 2004.

. et al. Comitês de ética em pesquisa: adequação à Resolução $\mathrm{n}$. 196/96. Rev. Assoc. Med. Bras., v. 50, n. 4, p. 457-462, 2004.

HOSSNE, W. S. Autonomia e pacientes psiquiátricos. Caderno de Ética em Pesquisa, n. 3, p. 12, 1999.

JUNGES, J. R. Ética e consentimento informado. Caderno de Ética em Pesquisa, n. 4, p. 22-23, 2000.

LOTT J. P. Module three: vulnerable/special participant population. Developing World Bioeth, v. 5, n. 1, p. 30-54, 2005.

LOWDEN, J. Children's rights: a decade of dispute. Journal of Advanced Nursing, v. 37, n. 1, p. 100-107, 2002.

MELO, A. C. R.; LIMA, V. M. Bioética: pesquisa em seres humanos e comitês de ética em pesquisa. Breves esclarecimentos. Rev. Digital, n. 78, 2004. Disponível em: <http://www.efdesportes.com/efd78/etica.htm>. Acesso em: 6.abr.2005.

NAIK, E.; KARPUR, A.; TAYLOR, R. et al. Rural indian tribal communities: an emerging high-risk group for HIVIAIDS. International Health and Human Rights, v. 5, n. 1, 2005. Disponível em: <http://www.biomedcentral.com/1472698X/5/1>. Acesso em: 8.jun.2005.

RESNIK, D. B. Research participation and financial inducements. The American Journal of Bioethics, v. 1, n. 2, p. 54-56, 2001.

RIIS, P. Thirty years of bioethics: the Helsinki Declaration 1964-2003. New Rev. Bioeth., v. 1, n. 1, p. 15-25, 2003.

ROGER, B. Research with protected population - vulnerable participants. Journal AAOHN, v. 53, n. 4, p. 156-157, 2005.

ROSSI, W. C.; REYNOLDS, W.; NELSON, R. M. Child assent parental permission in pediatric research. Theor. Med. Bioeth., v. 24, n. 2, p. 131-148, 2003.

SCHÜKLENK, U. Protecting the vulnerable: testing time for clinical research ethics. Social Science \& Medicine, v. 51, p. 969-977, 2000.

SEGRE, M. Autonomia e pacientes psiquiátricos. Caderno de Ética em Pesquisa, n. 3, p. 12, 1999.

ZOBILI, E. L. C. P.; FRACOLLI, L. A. Vulnerabilidade do sujeito de pesquisa. Caderno de Ética em Pesquisa, n. 8, p. 20-21, 2001. 